\title{
CONTRIBUIÇÕES DO MÉTODO PILATES SOBRE A ATENÇÃO E A APRENDIZAGEM DE ESTUDANTES COM BAIXO RENDIMENTO ESCOLAR
}

\author{
Anelise Corrêa Antonello \\ Universidade Federal do Pampa, Uruguaiana, Rio Grande do Sul, Brasil \\ Rachel Fernandes Medeiros \\ Universidade Federal do Pampa, Uruguaiana, Rio Grande do Sul, Brasil \\ Simone Lara \\ Universidade Federal do Pampa, Uruguaiana, Rio Grande do Sul, Brasil
}

\begin{abstract}
Resumo
O objetivo deste estudo foi avaliar os efeitos da prática de exercício físico, através do método Pilates, sobre os níveis de atenção e a aprendizagem de estudantes com baixo rendimento escolar. Foram selecionadas crianças com baixo rendimento escolar, para participar da prática de exercícios baseados no método Pilates. As crianças foram avaliadas antes e após a prática do método, através do teste de atenção (seletiva e alternada). Os professores responsáveis avaliaram as dificuldades na conduta, matemática, escrita, leitura, e rendimento escolar geral dos estudantes. Após a intervenção houve uma melhora dos níveis de atenção e uma redução nas dificuldades de aprendizagem, evidenciando a influência positiva do método Pilates nos estudantes com baixo rendimento escolar.
\end{abstract}

Palavras-Chaves: Estudantes. Aprendizagem. Atenção.

\section{Introdução}

A dificuldade de aprendizagem consiste em uma discrepância entre o que se espera que a criança tenha a capacidade de aprender e o que ela realmente aprende, pois seu potencial intelectual não corresponde com o esperado (Maschio e Sansão, 2013).

No âmbito escolar, vários são os fatores que podem estar associados ao desenvolvimento destas dificuldades. Assim, podem ser encontrados fatores intrínsecos (genéticos) e fatores extrínsecos (experiência) envolvidos nas dificuldades de aprendizagem e consequentemente no rendimento escolar. Entre os fatores intrínsecos, pode-se destacar as dislexias, discalculias, dispraxias, disgnosias, déficit de atenção e hiperatividade (Rotta e cols., 2005). Os fatores extrínsecos estão associados a problemas socioeconômicos, como condição de vida e desnutrição; afetivos, como estrutura familiar decadente, e ainda problemas no ambiente escolar, como por exemplo, as condições do corpo docente, a capacitação de professores e a não valorização do profissional (Rotta e cols., 2005).

Com relação à atenção, a mesma apresenta-se como um fenômeno pelo qual o ser humano processa ativamente uma quantidade limitada de informações do enorme montante disponível por meio dos órgãos dos sentidos, de memórias armazenadas e de outros processos cognitivos (Signoretti, 2011). Assim a atenção está em praticamente todas as ações e processos mentais humanos, mesmo que de forma inconsciente, portanto os níveis de atenção podem estar diretamente ligados a aprendizagem e, consequentemente, ao rendimento escolar 
satisfatório ou deficiente (Capovilla e Dias, 2008). Corroborando, um estudo identificou desordens importantes na atenção seletiva e na atenção alternada de estudantes com baixo rendimento escolar (Nunes e cols., 2014).

Ainda, há evidencias que as dificuldades de aprendizagem podem ter uma relação direta com o atraso no desenvolvimento motor (Rosa Neto e cols., 2007), uma vez que um desenvolvimento motor deficiente leva a um desenvolvimento intelectual pobre.

Observa-se que algumas escolas públicas do país não ofertam a disciplina de educação física no currículo dos anos iniciais do ensino fundamental. Nas mesmas, esta prática só tem início a partir do sexto ano, fato este extremamente negativo ao desenvolvimento potencial global da criança. Da mesma forma, este fato pode estar associado ao baixo rendimento escolar, implicando negativamente no desenvolvimento intelectual do estudante, sendo que um desenvolvimento motor pobre pode influenciar na dificuldade de aprendizagem. Ainda, a falta de estímulo motor, pela não aplicabilidade de nenhuma atividade física, pode prejudicar o aprendizado, os níveis de atenção, a leitura, escrita, matemática e linguagem dos alunos (Gregório e cols., 2002). Sob esta perspectiva, a inclusão de práticas relacionadas à atividade física e promoção à saúde são alternativas viáveis tanto para um melhor desenvolvimento motor quanto cognitivo.

O método Pilates pode ser uma alternativa para a implementação de exercícios físicos nesta população, e baseia-se em seis princípios básicos: concentração, controle, centro, respiração, precisão e fluidez (Latey, 2002). A concentração reflete a interação entre mente e corpo, onde ambos trabalham associados. O controle consiste no controle consciente de todos os movimentos musculares executados pelo corpo. $\mathrm{O}$ centro, conhecido como $\mathrm{O}$ "Powerhouse", é a essência do sistema muscular por representar a origem de todos os movimentos funcionais. A respiração está intimamente relacionada ao método, a fim de que o corpo receba quantidade necessária de oxigênio para um desempenho adequado. A precisão e a fluidez estão relacionadas à execução dos movimentos de forma controlada e coordenada, na qual os movimentos são realizados de forma harmônica e com leveza (Camarão, 2004; Muscolino e Cipriani, 2004; Costa e cols., 2012; Latey, 2002).

Por meio destes princípios, o método Pilates tem como finalidade o aumento do controle neuromuscular, da força muscular, do equilíbrio e consciência corporal, trabalhando o corpo como um todo, possibilitando assim uma maior integração do indivíduo em seu dia-adia (Camarão, 2004). O método Pilates é uma atividade física que se diferencia por trabalhar em conjunto com a concentração mental, enquanto outras atividades são realizadas de forma superficial e mecânica (Pilates, 2010). O método possui uma variedade ampla de exercícios que podem ser realizados no solo, utilizando a resistência do próprio corpo, e com acessórios, como bola e faixas elásticas, ou, em aparelhos dotados de molas e polias para promover a resistência (Siler, 2008).

Assim, a ampla gama de exercícios realizados, com instrumentos diferentes, torna o método Pilates uma atividade com um aspecto lúdico importante, que desafia e estimula as crianças a praticar, proporcionando, através de seus princípios, uma melhor percepção do seu próprio corpo. Contudo, seus efeitos em crianças com idade escolar ainda são escassos.

Com base nos princípios desse método, acredita-se que um programa de exercícios de Pilates possa acarretar na melhora da atenção e no rendimento dos estudantes, favorecendo a aprendizagem no contexto escolar. Logo, o objetivo deste estudo foi avaliar os efeitos do método Pilates sobre os níveis de atenção e a aprendizagem de estudantes com baixo rendimento escolar.

\section{Metodologia}


Trata-se de um estudo longitudinal, prospectivo e quantitativo, onde foram incluídos estudantes, regularmente matriculados em três turmas do $5^{\circ}$ ano do ensino fundamental, de uma escola pública, no município de Uruguaiana, interior do Rio Grande do Sul, Brasil. Esta instituição apresenta um baixo Índice de Desenvolvimento da Educação Básica (IDEB) e localiza-se em uma região periférica e de vulnerabilidade social no município. A mesma não apresenta a disciplina de educação física escolar no currículo dos anos iniciais.

O estudo ocorreu no período compreendido entre os meses de março a novembro de 2014. Os critérios de inclusão foram: crianças matriculadas no $5^{\circ}$ ano do ensino fundamental, que não praticavam exercício físico regular e educação física escolar, e que apresentavam baixo rendimento escolar, de acordo com a percepção do docente responsável. Entre os critérios de exclusão encontraram-se aqueles que apresentavam algum comprometimento físico que os impedissem de praticar o método Pilates.

O processo de seleção dos estudantes teve início através do contato com a direção da escola, onde foi solicitada a escolha de turmas de estudantes que apresentassem menores rendimentos escolares, sendo essas escolhidas pelos próprios professores, devido à percepção no convívio diário em sala de aula. Ainda, o critério utilizado pelos professores nesta seleção, foram aquelas turmas de estudantes que utilizavam com maior frequência a sala de recursos da escola, por apresentarem constantemente baixo rendimento ao longo do período letivo. Estes espaços consistem em locais destinados ao atendimento de estudantes que apresentam, dentre outras dificuldades, um baixo rendimento escolar, e assim, necessitam de um apoio pedagógico específico da escola, através da intervenção de professores que possuem formação adequada para esta função.

Após este contato inicial, foi realizada uma reunião com os responsáveis legais pelos estudantes, a fim de explicar os objetivos do estudo, e os mesmos deveriam assinar o termo de consentimento livre esclarecido (TCLE), consentindo a participação do menor no estudo. $\mathrm{O}$ projeto foi aprovado pelo Comitê de Ética e Pesquisa da Universidade Federal do Pampa (UNIPAMPA), sob o número de protocolo 457.088.

Após essa etapa, duas turmas de estudantes, totalizando 32 crianças, foram incluídas no estudo, e iniciaram a avaliação e a prática do método, integrando o grupo intervenção (GI). Contudo, houve perda amostral de oito estudantes, devido a não participação de no mínimo $75 \%$ de frequência ao longo do estudo, e por evasão escolar. Assim, participaram efetivamente do GI 24 estudantes, sendo 15 meninas e 09 meninos. A terceira turma selecionada não recebeu nenhuma intervenção com o método Pilates, e sim, palestras gerais sobre hábitos de vida saudáveis, integrando o grupo controle (GC), totalizando 14 estudantes, sendo 12 meninas e 02 meninos.

Os estudantes participaram de uma avaliação inicial, constando de: a) avaliação do nível de atenção; b) avaliação do rendimento escolar.

- A avaliação do nível de atenção: Para esta avaliação utilizou-se o teste de atenção por cancelamento (Montiel e Capovilla, 2007). Este teste consiste em três matrizes impressas com estímulos diferentes, em que a tarefa do indivíduo consiste em assinalar todos os estímulos iguais a um estimulo alvo pré- determinado. A primeira etapa do teste tem por objetivo a avaliação da atenção seletiva, que consiste na capacidade do indivíduo de atentar a um estímulo determinado dentre outros estímulos diferentes. A atenção seletiva refere-se à capacidade de emitir respostas a um estímulo específico desconsiderando aqueles não relevantes, mantendo sobre este estímulo específico uma orientação atencional focal. A segunda etapa a dificuldade para o indivíduo aumenta, porém o objetivo segue sendo o mesmo da primeira. A terceira etapa do teste é avaliada a atenção alternada, ou seja, capacidade do indivíduo de mudar o foco da atenção. A atenção alternada, por sua vez, referese à capacidade de substituir um estímulo-alvo da atenção por outro, por meio da investigação ativa no ambiente, identificando particularidades em estímulos mediante as alterações. $\mathrm{Na}$ 
realização do teste, o indivíduo tem um tempo máximo de 1 minuto para cada etapa (Capovilla e Dias, 2008). Neste estudo foram analisados os escores do número de acertos de cada etapa do teste, e o número total de acertos das três etapas.

- Avaliação do Rendimento Escolar: O rendimento escolar dos estudantes foi avaliado pelo professor responsável, por meio de um instrumento, considerando os itens: conduta, matemática, escrita, leitura e rendimento escolar geral (Amaro, 2010). Logo, o professor deveria assinalar SIM para os itens nos quais o estudante apresentava dificuldade de aprendizagem e NÃO quando esta dificuldade não estava presente.

O GI, que praticou o método Pilates, foi avaliado antes e após o estudo quanto aos níveis de atenção e ao rendimento escolar. O GC foi avaliado antes e após a intervenção quanto aos níveis de atenção. O protocolo de intervenção do GI foi praticado duas vezes por semana, com duração de uma hora, utilizando colchonetes e bolas. Foi constituído por três etapas modificadas, baseado no protocolo proposto por Araujo (2010), e adaptado pelos pesquisadores:

- Preparação (10 minutos): treino inicial de aprendizagem da respiração correta, indivíduos em decúbito dorsal, com as mãos posicionadas sobre o abdômen para um melhor feedback, no qual foi orientado pra a inspiração, puxar o ar pelo nariz e encher a barriga, seguida da expiração, soltando o ar pela boca e realizando a contração dos músculos do powerhouse, associado aos outros princípios do método. Seguidas por alongamentos musculares globais, envolvendo tronco e membros superiores e inferiores.

- Parte especifica (45 minutos): foram utilizados exercícios específicos do método Pilates (solo e com bola), sendo realizada uma série de seis repetições para cada exercício, cujas descrições e objetivos se encontram no quadro1 (Page, 2010).

- Encerramento (5 minutos): para finalizar as atividades, foram realizadas técnicas de relaxamento muscular.

Quadro 1. Protocolo de exercícios do método Pilates

\begin{tabular}{|c|c|c|}
\hline $\begin{array}{l}\text { Exercício do método } \\
\text { Pilates }\end{array}$ & Nível & Objetivo \\
\hline Mermaid & Básico & Alongar cadeia lateral de tronco \\
\hline Single leg kick & Básico & $\begin{array}{l}\text { Alongar quadríceps e abdominais } \\
\text { Fortalecer isquitibiais e paravertebrais }\end{array}$ \\
\hline $\begin{array}{l}\text { Bouncing while } \\
\text { kicking on the ball }\end{array}$ & Básico & Melhorar equilíbrio, coordenação neuromuscular \\
\hline Bridging on the ball & $\begin{array}{l}\text { Básico à } \\
\text { intermediário }\end{array}$ & $\begin{array}{l}\text { Fortalecer abdominais e paravertebrais. } \\
\text { Melhorar a coordenação neuromuscular }\end{array}$ \\
\hline Rollup & $\begin{array}{l}\text { Básico à } \\
\text { intermediário }\end{array}$ & $\begin{array}{l}\text { Alongar cadeia muscular posterior } \\
\text { Fortalecer flexores de quadril }\end{array}$ \\
\hline Spine twist & Intermediário & $\begin{array}{l}\text { Aumentar a mobilidade em rotação da coluna vertebral } \\
\text { Estabilizar a pelve }\end{array}$ \\
\hline Saw & Intermediário & $\begin{array}{l}\text { Aumentar a mobilidade em rotação e inclinação da coluna } \\
\text { vertebral }\end{array}$ \\
\hline Swan on the Ball & Intermediário & Fortalecer de paravertebrais e coordenação \\
\hline Push-Up on the Ball & $\begin{array}{l}\text { Intermediário à } \\
\text { avançado }\end{array}$ & Fortalecer músculos dos membros superiores \\
\hline Scissors & Avançado & $\begin{array}{l}\text { Fortalecer abdominais e Alongar cadeia muscular } \\
\text { posterior }\end{array}$ \\
\hline
\end{tabular}

Fonte: Page, 2010 
A figura 1 ilustra a prática de alguns exercícios do método, descritos no quadro 1, sendo: Spine twist, Push-Uponthe Ball e Bridging on the Ball (posição inicial e final do exercício).

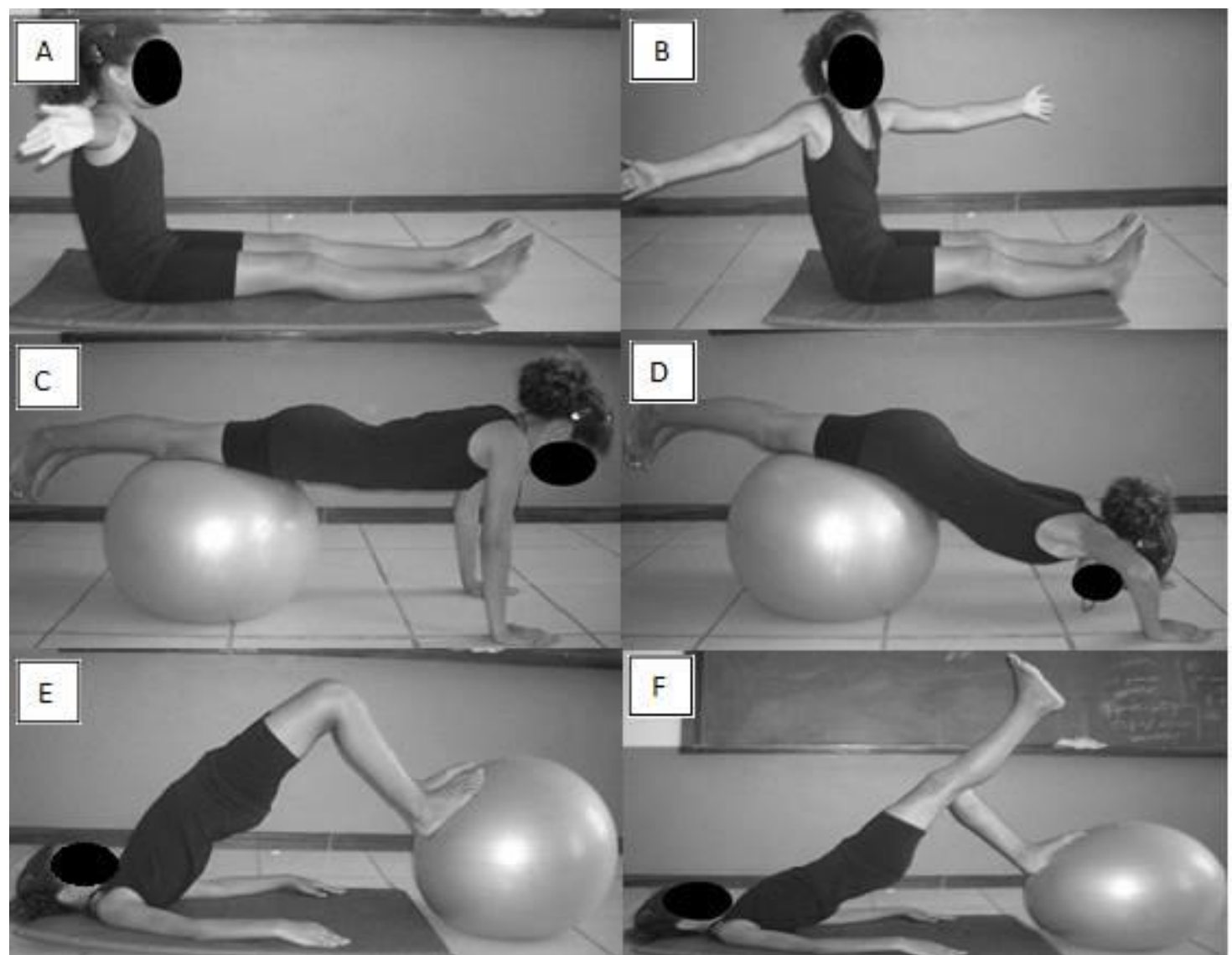

Figura 1. Prática do método Pilates: Spine twist (1A. Posição inicial, 1B Posição final); PushUp on the Ball (1C Posição inicial, 1D Posição final) e Bridging on the Ball (1E. Posição inicial, 1F Posição final). Fonte: os autores, 2014.

Os dados foram apresentados como média \pm desvio padrão ou frequência absoluta e relativa. A normalidade dos dados foi confirmada por meio do teste de Kolmogorov-Smirnov. $\mathrm{O}$ teste $\mathrm{t}$ de student pareado foi utilizado para analisar as variáveis de interesse do estudo no mesmo grupo, entre os períodos pré e pós-intervenção e o teste $t$ de student não pareado para comparação inter-grupos. O teste exato de Fisher foi utilizado para determinar as diferenças entre as proporções de respostas do rendimento escolar dos estudantes do grupo intervenção. Foi considerado como estatisticamente significativo um valor de $\mathrm{p}<0,05$. Para a obtenção das análises o software STATA versão 14.1 foi utilizado.

\section{Resultados}

Foram incluídas três turmas cursando o quinto ano do ensino fundamental, totalizando 24 escolares no GI e 16 escolares no GC, com idade entre 10 e 14 anos.

A tabela 1 demonstra o nível de atenção dos estudantes do GI e GC pré e pósintervenção, na comparação intra-grupos, considerando que o GI praticou o método Pilates e o GC participou apenas de atividades globais em saúde. São indicados os valores de referência/número máximo de acertos, o número de acertos dos estudantes em cada etapa do 
teste, bem como o número total de acertos nas três etapas do teste. Assim, quanto mais próximo ao valor de referência/número máximo de acertos, maior é o número de acertos no teste, e, portanto, melhor é o nível de atenção do estudante.

Foi possível verificar, após a intervenção, que houve um aumento significativo do número de acertos nas três etapas do teste nos estudantes do GI. Já no GC, houve um aumento significativo de acertos na $1^{\mathrm{a}}$ e na $2^{\mathrm{a}}$ etapa, o que não ocorreu com $3^{\mathrm{a}}$ a etapa do teste.

Tabela 1: Nível de atenção dos Estudantes Pré e Pós-Intervenção

GC: grupo controle, GI: grupo Pilates; *indicam valores significativos $(\mathrm{p}<0,05)$

\begin{tabular}{|c|c|c|c|c|c|c|c|}
\hline Acertos & $\begin{array}{c}\text { Valores de } \\
\text { referência / } \\
\text { número } \\
\text { máximo de } \\
\text { acertos }\end{array}$ & $\begin{array}{l}\text { GC } \\
\text { Pré }\end{array}$ & $\begin{array}{l}\text { GC } \\
\text { Pós }\end{array}$ & $\mathbf{P}$ & $\begin{array}{l}\text { GI } \\
\text { pré }\end{array}$ & $\begin{array}{l}\text { GI } \\
\text { Pós }\end{array}$ & $\mathbf{p}$ \\
\hline $\begin{array}{ll}\text { Número } & \text { de } \\
\text { acertos na } & 1^{\text {a }} \\
\text { etapa } & \end{array}$ & 50 & $36,28 \pm 7,7$ & $45,21 \pm 5,26$ & $0.0008^{*}$ & $34,30 \pm 8,18$ & $45.269 \pm 5,04$ & $0,0001 *$ \\
\hline $\begin{array}{ll}\text { Número } & \text { de } \\
\text { acertos na } & 2^{\text {a }} \\
\text { etapa }\end{array}$ & 7 & $2,50 \pm 1,01$ & $3,50 \pm 1,28$ & $0,03^{*}$ & $2,30 \pm 1,0$ & $3,92 \pm 2,0$ & $0,001 *$ \\
\hline $\begin{array}{l}\text { Número de } \\
\text { acertos na } 3^{\text {a }} \\
\text { etapa }\end{array}$ & 52 & $36,35 \pm 6,80$ & $38,14 \pm 7,66$ & 0,40 & $28,92 \pm 11,29$ & $44,71 \pm 17,52$ & $0,002 *$ \\
\hline $\begin{array}{l}\text { Total do } \\
\text { número de } \\
\text { acertos nas } \\
\text { três etapas }\end{array}$ & 109 & $75,28 \pm 13,08$ & $86,85 \pm 11,83$ & $0.0032^{*}$ & $67,69 \pm 13,69$ & $89,80 \pm 14,69$ & $0.0001^{*}$ \\
\hline
\end{tabular}

Na comparação inter-grupos (entre GI e GC), relativa ao número de acertos nas três etapas do teste, não houveram diferenças significativas, tanto pré quanto pós- intervenção. Esta análise evidencia que não houve diferença dos níveis de atenção entre os grupos, tanto antes, quanto após o estudo.

O rendimento escolar dos 24 estudantes do GI foi avaliado pelo professor pré e pósintervenção (tabela 2). Os dados evidenciaram uma redução significativa nas dificuldades associadas à escrita, à leitura e ao rendimento escolar dos estudantes. Com relação à conduta e à matemática, não houve modificação significativa ao longo da intervenção. 
Tabela 2. Dificuldades de aprendizagem dos estudantes

\begin{tabular}{lccc}
\hline Dificuldade & GI PRÉ & GI PÓS & P \\
\hline Conduta & $2(8,4 \%)$ & $2(8,4 \%)$ & $\mathrm{P}=1,39$ \\
Sim & $22(91,6 \%)$ & $22(91,6 \%)$ & \\
Não & & & \\
Matemática & $7(29,17)$ & $2(8,4 \%)$ & $\mathrm{P}=0,13$ \\
Sim & $17(70,83 \%)$ & $22(91,6 \%)$ & \\
Não & & & \\
Escrita* & $9(37,5 \%)$ & $2(8,4 \%)$ & \\
Sim & $15(62,5 \%)$ & $22(91,6 \%)$ & $\mathrm{P}=0,03$ \\
Não & & & \\
Leitura* & $6(25 \%)$ & $0(0 \%)$ & \\
Sim & $18(75 \%)$ & $24(100 \%)$ & $\mathrm{P}=0,04$ \\
Não & & $0(0 \%)$ & \\
Rendimento escolar* & $5(20,9 \%)$ & $24(100 \%)$ & \\
Sim & $19(79,1 \%)$ & & \\
Não & & &
\end{tabular}

GI: grupo Pilates; *indicam valores significativos $(\mathrm{p}<0,05)$

\section{Discussão}

O presente estudo demonstrou que a prática de exercício físico regular, por meio do método Pilates, esteve associada há uma melhora nos níveis de atenção (tanto seletiva, quanto alternada) de estudantes com baixo rendimento escolar. Contudo, nas crianças que não praticaram esta modalidade, foi observada melhora apenas na atenção seletiva $\left(1^{\circ}\right.$ e $2^{\circ}$ etapa), o que não ocorreu sobre a atenção alternada ( $3^{\circ}$ etapa). Este fato evidenciou um possível benefício do método Pilates, especialmente quanto à capacidade do estudante alterar sua atenção de acordo com um novo estímulo. De fato, esta é uma função mais complexa, pois depende principalmente da memória de trabalho e do controle neural (Pinto, 2006).

Nesse sentido, é conhecido que a prática de exercício físico tem um efeito positivo sobre as funções cognitivas como memória, atenção e raciocínio (Laurine e cols., 2001). O estudo de Abramovitch e cols. (2013) demonstrou correlação positiva entre a prática de exercício físico e a melhora de sintomas relacionados aos transtornos de atenção. Nesta perspectiva, um estudo encontrou que, em 224 estudantes com média de 12 anos de idade, houve uma associação positiva entre maiores níveis de exercício físico com melhores padrões de atenção (Syväoja e cols., 2014). O trabalho ainda evidenciou que quanto maior o tempo de inatividade física, em atividades frente ao computador e ao vídeo-game, piores são as funções cognitivas destas crianças.

Barbosa (2008) evidenciou em seu trabalho, que meninos que treinavam regularmente em um centro de formação de futebol, na faixa-etária de 10 e 11 anos de idade, obtiveram um maior número de acertos no teste de cancelamento, quando comparados aos que praticavam somente aulas de educação física escolar. Assim, observa-se que a qualidade da prática de exercício físico tem influência positiva sobre o desenvolvimento das habilidades atencionais em crianças. 
O achado de Barbosa (2008) vai ao encontro dos dados do presente estudo, na qual foi evidenciado um melhor nível de atenção alternada nos praticantes do método Pilates, quando comparado aos não praticantes. Assim, nota-se que a qualidade do exercício praticado, por meio dos princípios do método Pilates, interferiu positivamente sobre a habilidade atencional dos estudantes. De fato, para realizar os exercícios do método corretamente, com qualidade, fluidez e destreza, é necessário direcionar a concentração e a atenção para a execução de todas as atividades. Ainda, os movimentos devem ser realizados de forma precisa, portanto é necessário concentração para realizá-los de forma adequada, devendo manter a correta colocação das partes do corpo, o que está intimamente relacionada com a postura e consciência corporal. Ademais, o estudante necessita de atenção para associar o controle dos movimentos à respiração, princípios estes presentes em todos os movimentos do método.

Os estudantes que praticaram o método Pilates no presente estudo obtiveram uma melhora significativa no desempenho escolar, especialmente nos aspectos relacionados com a escrita, a leitura e ao rendimento escolar, de acordo com a percepção do professor. Corroborando, o trabalho de Spitzer e Hollmann (2013) avaliou os efeitos da prática de uma modalidade de exercício físico regular sobre o desempenho escolar de estudantes (através da percepção do professor), e sobre os níveis de atenção (através de um teste de concentração). Assim, após a intervenção, foram encontrados efeitos positivos sobre as duas variáveis avaliadas, evidenciando melhora na atenção e no rendimento escolar dos estudantes.

Contudo, cabe ressaltar que os efeitos do exercício físico sobre a aprendizagem dependem do tempo e da frequência com que o mesmo é realizado. No estudo de Ahamed e cols. (2007) avaliaram os efeitos da inclusão de quinze minutos de exercício físico diário por um ano, sobre o desempenho escolar de alunos, relacionados às áreas específicas, como matemática, leitura e linguagem. Após a intervenção, os autores não evidenciaram nenhuma alteração sobre o desempenho acadêmico dos estudantes. Este resultado pode ser justificado pelo curto tempo de acréscimo de atividade física praticado, não sendo considerável para a melhora dos parâmetros de desempenho escolar. Ericsson e Karlsson (2014) encontraram que o desempenho escolar, na língua inglesa e na matemática, bem como as habilidades motoras, foram superiores em estudantes que praticavam exercício físico com uma frequência de cinco vezes semanais, quando comparados aos que realizavam as atividades apenas duas vezes semanais, demonstrando o efeito importante da frequência da prática de exercício físico em estudantes. Além disso, o estudo evidenciou que alunos que apresentaram um déficit nas habilidades motoras, apresentaram também dificuldades no desempenho escolar.

De fato, os ganhos obtidos sobre o rendimento escolar, especialmente relacionado à escrita, nos praticantes do método Pilates no presente estudo, podem estar atrelados ao ganho de habilidades motoras, que são trabalhadas por meio do método. Ou seja, desenvolvendo a motricidade e o controle dos movimentos, o mesmo pode melhorar a qualidade da escrita, relacionado ao movimento fino manual para escrever. Segundo Calvo e cols. (2014), o ato de escrever requer um alto nível de coordenação e controle motor, e o movimento da escrita é produzido por mudanças na coordenação das estruturas mais distais. Nesse contexto, autores retratam que o método Pilates melhora a simetria e o alinhamento corporal, postura e desenvolvimento motor, o que pode levar há um aperfeiçoamento das habilidades motoras (Siler, 2008; Panelli e Marco, 2009).

Vale salientar que, a melhora nos níveis de atenção, que constitui um dos princípios do método, pode estar diretamente relacionada com a melhora no rendimento escolar dos estudantes do atual estudo, pois, uma melhor demanda de atenção, pode acarretar em um melhor desempenho nas atividades escolares. Nesse sentido, Siqueira \& Giannetti (2011) relatam que a atenção tem papel essencial na aquisição de novas habilidades (aprendizagem), onde, através da atenção, se filtram as informações relevantes no meio (atenção seletiva) e se mantém, sob foco, esta informação desejada (atenção sustentada e focalizada). Logo, de 
acordo com esse estudo, melhores níveis de atenção facilitam a aprendizagem, fato este que pode repercutir positivamente no ambiente escolar. Ainda, no contexto do estudo, o método Pilates influenciou positivamente os níveis de atenção dos estudantes, o que pode ter repercussão direta também sobre a aprendizagem escolar.

Cabe ressaltar, contudo, que o estudo avaliou os estudantes sob uma perspectiva longitudinal, ou seja, além do efeito da prática do exercício físico, os ganhos adquiridos sobre a atenção e o rendimento escolar também se devem ao próprio crescimento e à maturação fisiológica das crianças, e demais estímulos que elas receberam ao longo desse tempo. Corroborando, autores retratam que o desenvolvimento é entendido como uma interação entre as características biológicas individuais (crescimento e maturação) e o meio ambiente ao qual o sujeito é exposto durante a vida (Frisancho, 2009; Malina e cols., 2009), ou seja, ambos os fatores influenciam sobre o desenvolvimento global da criança. Contudo, a experiência, juntamente com a maturação e crescimento, irá propiciar significativamente o desenvolvimento de várias capacidades físicas e melhoria no desempenho motor (Sá e cols., 2014). De acordo com Rosa Neto e cols. (2010), o fato de a criança explorar o ambiente por meio de atividades motoras (como o exercício físico) implicará em modificações no seu desenvolvimento físico, perceptivo-motor, moral e afetivo. Logo, apesar de considerar os efeitos biológicos, inerentes ao processo de crescimento das crianças, a importância do fator ambiental, relativo à prática do método Pilates no presente estudo, se mostrou relevante sobre os ganhos adquiridos pelos estudantes.

A limitação do estudo está relacionada à perda amostral ao longo da intervenção, e a não avaliação do rendimento escolar pelos alunos do GC. Apesar dos estudos indicarem a associação positiva entre a prática de exercício físico, a atenção e o rendimento escolar, não há estudos relacionando especificamente o método Pilates com a melhora destas variáveis em estudantes. Sendo assim, se fazem necessários maiores estudos para elucidar os efeitos do método sobre estas variáveis no contexto escolar.

\title{
Considerações Finais
}

Através da prática de exercícios baseados no método Pilates, foi evidenciado uma influência positiva nos níveis de atenção e no rendimento escolar de estudantes com baixo rendimento escolar. Assim, conclui-se que o método contribuiu de forma importante sobre as variáveis analisadas, e pode ser uma ferramenta relevante para promover a saúde de estudantes nos anos iniciais, melhorando o seu desempenho no contexto escolar.

Contudo, considerando a escassez de dados envolvendo a prática do método Pilates na escola, especialmente sobre os níveis de atenção e desempenho escolar de estudantes, torna-se necessária a realização de maiores estudos na área.

\section{PILATES METHOD CONTRIBUTIONS ON ATTENTION AND LEARNING OF STUDENTS WITH LOW SCHOOL PERFORMANCE}

\begin{abstract}
The aim of this study was to assess the effects of physical exercise, through the Pilates method, based on the levels of care and learning of students with poor academic performance. Poor academic performance children were selected to participate in the practice of exercises based on the Pilates method. The children were evaluated before and after practicing the method, through the attention test (selective and alternating). The responsible staff members evaluated the difficulties in behavior, math, writing, reading, and overall school performance. After the intervention, there was nain provement of the attention levels and on the reduction
\end{abstract}


of the learning disabilities, showing the positive influence of Pilates method in students with poor academic performance.

Keywords: Students. Learning. Attention.

\title{
CONTRIBUCIONES DE LO METODO PILATES EN ATENCIÓN Y ESTUDIANTES APRENDIENDO CON LA ESCUELA DE BAJOS INGRESOS
}

\begin{abstract}
Resumen
El objetivo de este estúdio fue evaluar los efectos de la práctica de ejercicio físico, a través del método Pilates, sobre los niveles de atención y aprendizaje de estudiantes com bajo rendimiento escolar. Fueron selecionados niños com bajo rendimiento escolar, para participar em La práctica de ejercicios basados enel método Pilates. Los niños fueron evaluados antes y después de La práctica del método, a través del test de atención. Los profesores responsables evaluaron la dificultad em la conducta, matemática, escritura, lectura y el rendimiento escolar general de los estudiantes. Hubo una mejora em los niveles de atención y uma reducción em lãs dificultades de aprendizaje, evidenciando La influencia positiva del método Pilates em los estudiantes com bajo rendimiento escolar.
\end{abstract}

|

Palabras claves: Estudiantes. Aprendizaje. Atención.

\section{Referências}

ABRAMOVITCH, A.; GOLDZWEIG, G.; SCHWEIGER, A. Correlates of Physical Activity with Intrusive Thoughts, Worry and Impulsivity in Adults with Attention Deficit/Hyperactivity Disorder: A Cross-sectional Pilot Study. The Israel J psych and related scien, v.50, n. 1, p. 47-53, 2013.

AMARO, K. N. Intervenção motora para escolares com dificuldades na aprendizagem. 2010. 114f. Dissertação (Mestrado em Ciências do Movimento Humano) - Centro de Ciências da Saúde e do Esporte da Universidade do Estado de Santa Catarina Universidade do estado de Santa Catarina, UDESC, Florianópolis-SC, 2010.

AHAMED, Y.; MACDONALD, H.; REED, K.; NAYLOR, P. J.; LIU-AMBROSE, T.; MCKAY, H. School-based physical activity does not compromise children's academic performance. MedSci Sports Exerc., v. 39, n. 2, p. 371-6, 2007.

ARAÚJO, M. E. A.; SILVA, DA B. E.; VIERA, P. C.; CADER, S. A,.;MELLO, DE D. B.; DANTAS, E. H. M. Redução da dor crônica associada à escoliose não estrutural, em universitárias submetidas ao método Pilates. Motriz, v.16, n. 4, p. 958-966, 2010.

BARBOSA, C. Avaliação de atenção e habilidades motoras em atletas de 10-17 anos. 2008. Tese de doutorado, Universidade São Francisco, Itatiba-SP, 2008.

CALVO, A.; PACUlLIB, A.; HIRAGAC, C.; MASSOD, M.; PELLEGRINIC. A. Qualidade da escrita: Intervenção baseada na variação da produção de força dos dedos. Cad. Ter. Ocup. UFSCar, São Carlos, v. 22, n. 2, p. 317-325, 2014.

CAMARÃO, T. Pilates no brasil corpo e movimento. $2^{\circ}$ edição. Rio de janeiro: Elsevier, 2004. 
CAPOVILlA, A. G. S.; DIAS, N. M. Desenvolvimento de habilidades atencionais em estudantes da $1^{\mathrm{a}}$ à $4^{\mathrm{a}}$ série do ensino fundamental e relação com o rendimento escolar. Revista Psicopedagogia, v. 25, n. 78, p. 198-211, 2008.

COSTA, L. M. R. D.; ROTH, A.; NORONHA, D. M. O método pilates no Brasil: uma revisão de literatura. Arquivos Catarinenses de Medicina, v.41, n. 3, p. 87-92, 2012.

ERICSSON, I.; KARLSSON, M. K. Motor skills and school performance in children with daily physical education in school - a 9 year intervention study. Scandinavian Journal of Medicine \& Science in Sports, v. 24, n. 2, p. 273-8, 2014.

FRISANCHO, A. R. Developmental adaptation: Where we go from here. American Journal of Human Biology, v. 21, p. 694-703, 2009.

GREGÓRIO, C. S. B.; PINHEIRO, E. C. T.; CAMPOS, D. E. O.; ALFARO, E. J. Evolução neuromotora de um recém-nascido pré-termo e a correlação com os fatores perinatais. Fisioterapia Brasil, v. 3, n. 4, p. 250-257, 2002.

LATEY, P. Updating the principles of the Pilates method. Part 2. Journal of Bodywork and Movement Therapies, v. 6, n. 2, p. 94-101, 2002.

LAURIN D.; VERREAULT, R.; LINDSAY, J.; MACPHERSON, K.; ROCKWOOD, K. Physical activity and risk of cognitive impairment and dementia in elderly persons. Archives of Neurology, v. 58, n. 3, p. 498-504, 2001.

MALINA, R. M.; BOUCHARD, C.; BAR-OR, O. Crescimento, maturação e atividade física. São Paulo: Phorte, 2009.

MASCHIO, F. S.; SANSÃO, B. Z. Dificuldade de aprendizagem. UNIASSELV. Revista Maiêutica pedagogia, v. 01, n. 01, 2013.

MONTIEL, J. M.; CAPOVILLA, A. G. S. Teste de atenção por cancelamento. In: CAPOVIlla A. G. S.; CAPOVILlA F. C. Teoria e pesquisa em avaliação neuropsicológica. São Paulo: Memnon, p.119-24, 2007.

MUSCOLINO, J. E.; CIPRIANI, S. Pilates and the "powerhouse'. Journal of Bodywork and Movement Therapies, v. 8, p. 122-130, 2004.

ROSA NETO, F.; ALMEIDA, G. M. F. D.; CAON, G.; RIBEIRO, J.; CARAM, J. A.; PIUCCO, E. C. Desenvolvimento Motor de Crianças com Indicadores de Dificuldades na Aprendizagem Escolar. Revista brasileira Ciência e Movimento, v 15, n. 1, p. 45-51, 2007.

ROSA NETO, F.; SANTOS, A. P. M.; XAVIER, R. F. C.; AMARO, K. N. A Importância da avaliação motora em escolares: análise da confiabilidade da Escala de Desenvolvimento Motor. Revista Brasileira de Cineantropometria e Desempenho Humano, v. 12, n. 6, p. 422-427, 2010.

NUNES, L. C.; NEVES, D.; TEODÓSIO, G. DE F.; FLORIANO, P. M.; LARA, S. Perfil de estudantes dos anos iniciais com baixo rendimento escolar: importância da educação física na escola. Revista Brasileira Ciências e Movimento, v.22, n. 2, p. 36-46, 2014. 
PAGE P. Pilates Illustrated: Strength, flexibility, posture e balance. $1^{\circ}$ edição. Champaign, IL: Human Kinetics Publishers, 2010.

PANELLI, C.; MARCO, D. M. Método pilates e condicionamento do corpo, um programa para toda a vida. $2^{\circ}$ edição. São Paulo: Phorte, 2009.

PILATES, J. H. A obra completa de Joseph Pilates: Sua saúde, o retorno a vida pela contrologia. São Paulo: Phorte, 2010.

PINTO, K. O. Introdução à investigação neuropsicológica. Curso ministrado no II Congresso Brasileiro Psicologia: Ciência e Profissão, São Paulo: Conselho Federal de Psicologia, 2006.

ROTTA, N. T.; OHLWEILER, L.; RIESGO, D. S. R. Transtornos da Aprendizagem: Abordagem Neurobiológica e Multidisciplinar. Porto Alegre: Artmed, 2005.

SÁ, C. S. C.; CARVAlHO, B.; MAZZITELli, C. Equilíbrio e Coordenação Motora em Escolares Praticantes e Não Praticantes de Atividades Física e/ou Lúdica Extra-Escolar. Revista Neurociências, v.22, n. 1, p. 29-36, 2014.

SIGNORETTI, M. Z. C. Aspectos cognitivos, inteligência e atenção, do condutor e sua relação com infrações de trânsito. 2011. 66f. Monografia (Curso de Pós-graduação lato sensu em Psicologia de Trânsito), Centro Universitário de Araras, Araras/SP, 2011.

SILER, R. O corpo pilates: Um guia para o fortalecimento, alongamento e tonificação sem uso de maquinas.São Paulo: Summus, 2008.

SPITZER, U. S.; HOLLMANN, W. Experimental observations of the effects of physical exercise on attention, academic and prosocial performance in school settings. Thends in Neuroscience and Education, v. 2, n.1, p. 1-6, 2013.

SIQUEIRA, C. M.; GIANNETTI, J. M. Mau desempenho escolar: uma visão atual. Revista da Associação Médica Brasileira, v. 57, n. 1, p. 78-87, 2011.

SYVÄOJA, H. J.; TAMMELIN, T. H.; AHONEN, T.; KANKAANPÄ̈̈, A.; MARKO T.; KANTOMAA, M. T. Associations of Objectively Measured Physical Activity and Sedentary Time with Cognitive Functions in School-Aged Children. PLOS ONE, v. 9, n. 7, p. 1-10, 2014.

Recebido em: 31/01/2015

Revisado em: 16/10/2015

Aprovado em: 10/08/2016

Endereço para correspondência:

simonelara@ unipampa.edu.br

Simone Lara

Universidade Federal do Pampa.

Campus Uruguaiana - BR 472 KM 592

97500-970 - Uruguaiana, RS - Brasil 